
\title{
R Research Soure \\ Outcome of COVID-19 with Co-Existing Surgical Emergencies in Children: Our Initial Experiences and Recommendations
}

\author{
Samiul Hasan ( $\nabla$ samiulo45@gmail.com ) \\ Dhaka Shishu Hospital https://orcid.org/0000-0001-5470-2241 \\ Md Ayub Ali \\ Dhaka Shishu (Children) Hospital \\ Umama Huq \\ Bangladesh Institute of Child Health
}

Research article

Keywords: COVID 19, COVID 19 in children, Children's Surgery, Surgical emergency, Surgery in COVID 19 positive patients

Posted Date: September 18th, 2020

DOl: https://doi.org/10.21203/rs.3.rs-71589/v1

License: (a) (i) This work is licensed under a Creative Commons Attribution 4.0 International License. Read Full License 


\section{Abstract}

Background: COVID-19 has changed the practice of surgery vividly all over the world. This has already lead to a huge burden of rescheduled pediatric surgical cases worldwide. Though children are less likely to be infected and suffer less when infected, there is a growing fear among health care workers of being selfinfected, which is limiting the surgical care of children globally. This study aims to share our experiences with the outcome of COVID-19 in children who had a co-existing surgical emergency, which might help the pediatric surgeons globally to mitigate the effect of COVID 19 on pediatric surgery.

Methods: This is a retrospective observational study. We reviewed the epidemiological, clinical, and laboratory data of all patients admitted in our surgery department through the emergency department and later diagnosed to have COVID-19 by RT-PCR. During April 2020 - June 2020. A nasopharyngeal swab was taken from all patients irrespective of symptoms to detect SARS CoV 2 by RT-PCR to identify and isolate asymptomatic patients and patients with atypical symptoms. We divided the test positive patients into 4 age groups for the convenience of data analysis. Data were retrieved from hospital records and analyzed using SPSS (version 25) software. Ethical permission was taken from the hospital ethical review board.

Results: Total patients were 32. Seven (21.9\%) of them were neonates. Twenty-four (75\%) patients were male. The predominant diagnosis was acute abdomen followed by infantile hypertrophic pyloric stenosis (IHPS), myelomeningocele, and intussusception. Only two patients had mild respiratory symptoms (dry cough). Fever was present in $13(40.6 \%)$ patients. Fourteen (43.8\%) patients required surgical treatment. The mean duration of hospital stay was 5.5 days. One neonate with ARM died in the post-operative ward due to cardiac arrest. No patient had hypoxemia or organ failure. Seven health care workers $(5.51 \%)$ including doctors \& nurses got infected with SARS Co V2 during this period.

Conclusion: Our study has revealed a milder course of COVID-19 in children with minimal infectivity even when present in association with emergency surgical conditions. This might encourage a gradual restart to mitigate the impact of COVID-19 on children's surgery.

\section{Introduction:}

COVID-19 storm is showing its lethal face around the world and it is still beyond prediction how long it will continue, and how deadly it could be. Increasingly more people are being affected and people with coexisting diseases are suffering more.[1] The causative agent severe acute respiratory syndrome coronavirus 2 (SARS CoV 2) spreading rapidly around the world. As of 23rd July, more than 15 million people got infected with SARS CoV 2 and more than 6 million people have lost their lives because of COVID-19.[2]

Fortunately, the infection rate in children is low in comparison to adult and most cases are mild or asymptomatic. Therefore, a detailed clinical picture of COVID-19 in children is yet to come. Moreover, presentations of COVID-19 in children are more inconsistent than adults. Only around $25 \%$ of children present with common respiratory symptoms which leads to delay in diagnosis \& treatment and increases the risk of transmission.[3-6] 
As an effort to control the rapid transmission, governments \& organizations around the world have implemented distancing strategies. To comply with this strategy, healthcare institutions have taken several measures like discouraging hospital visits for non-emergency problems, prioritization, and re-scheduling health problems according to their time sensitivity. Pediatric surgical service is also not an exception. Different hospitals and associations have formalized protocols to ensure essential surgical service for children in need and at the same time to limit the spread of the virus and also to protect the health care workers.[7-10]

At the beginning of the outbreak of SARS CoV 2 in March in our country, we have also canceled all routine surgeries for children and have been performing only emergency surgeries to limit the spread of the SARS CoV 2. Though children experience a milder course of the disease, a growing fear of being infected among the health care workers preventing full phased activity in pediatric surgery. This study aims to illustrate the outcomes we observed in children with COVID-19 who had co-existing surgical emergencies. This will give a new insight to the pediatric surgeons around the world regarding the management of surgical problems of children during this COVID 19 pandemic.

\section{Methods:}

This is a retrospective observational study. We reviewed the epidemiological, clinical, and laboratory data of all patients admitted in our surgery department through the emergency department and later diagnosed to have COVID-19 by RT-PCR. The study duration was 3 months (April 2020 - June 2020).

In this study period, only children with emergency symptoms were admitted. A nasopharyngeal swab was taken from all patients irrespective of symptoms to detect SARS CoV 2 by RT-PCR to identify and isolate asymptomatic patients and patients with atypical symptoms. Emergency surgical services were provided immediately without delay and patients with positive test results were isolated according to the hospital protocol. We divided the test positive patients into 4 age groups for the convenience of data analysis.

Data were retrieved from hospital records and analyzed using SPSS (version 25) software. Categorical data were presented as frequency and numerical data were presented as mean \pm SD. Ethical permission was taken from the hospital ethical review board.

\section{Results:}

Total 32 children were tested positive for SARS CoV 2 by RT-PCR. Seven $(21.9 \%)$ of them were neonates (Table 1). The smallest one tested positive was 3 days old. Twenty-four $(75 \%)$ patients were male. The predominant diagnosis was acute abdomen followed by infantile hypertrophic pyloric stenosis (IHPS), myelomeningocele, and intussusception (Fig. 1). Only 2 patients had mild respiratory symptoms (dry cough). Fever was present in $13(40.6 \%)$ patients.[Supplemental material] 
Table 1

Age distribution of the patients.

\begin{tabular}{|c|c|c|c|c|c|}
\hline \multicolumn{6}{|c|}{ Age group } \\
\hline & & Frequency & Percent & Valid Percent & Cumulative Percent \\
\hline \multirow[t]{5}{*}{ Valid } & $<1$ month & 7 & 21.9 & 21.9 & 21.9 \\
\hline & 1month-12months & 11 & 34.4 & 34.4 & 56.3 \\
\hline & $>1$ Year - 5 Year & 5 & 15.6 & 15.6 & 71.9 \\
\hline & $>5$ Year & 9 & 28.1 & 28.1 & 100.0 \\
\hline & Total & 32 & 100.0 & 100.0 & \\
\hline
\end{tabular}

Apart from only one patient, none had any radiological evidence of COVID-19. Surgical treatment was provided to 14 (43.8\%) patients (Table 2). The mean duration of hospital stay was $5.5 \pm 1.6$ days.

[Supplemental material] One neonate with anorectal malformation (ARM) died in post-operative ward due to cardiac arrest; other patients were discharged after managing surgical symptoms with advice for home isolation. No patient had hypoxemia or organ failure. Seven health care workers out of $127(5.51 \%)$ including doctors \& nurses got infected with SARS Co V2 during this period. All of them were mildly symptomatic, did not require hospital admission, and recovered smoothly.

Table 2

Modalities of treatment.

\begin{tabular}{|c|c|c|c|c|c|}
\hline \multicolumn{6}{|c|}{ Modality of treatment: Surgical or non-surgical } \\
\hline & & Frequency & Percent & Valid Percent & Cumulative Percent \\
\hline \multirow[t]{3}{*}{ Valid } & Surgical & 14 & 43.8 & 43.8 & 43.8 \\
\hline & Non-surgical & 18 & 56.3 & 56.3 & 100.0 \\
\hline & Total & 32 & 100.0 & 100.0 & \\
\hline
\end{tabular}

\section{Discussion:}

COVID-19 has changed the way of surgical practice globally. This is a critical and unprecedented period, but efforts are being made to ensure inevitable emergency surgical care. The system is also adapting itself as more evidence is being generated.[7-11] From the beginning of the outbreak in our country, we have adopted the protocol to continue only the emergency admission at our department and try conservative treatment approaches.

More than $20 \%$ of our patients were neonates. The mode of transmission in these patients was not transparent. This might be due to the parents' tendency to hide history or reluctance to test when asymptomatic or mildly symptomatic. Vertical transmission of SARS CoV2 from mother to baby is not been proven yet. An increased level of virus-specific IgM has been reported in neonates of the infected mother, 
but this remains inconclusive due to the high rate of false positivity. Transmission through breast milk has also not been confirmed. Breast milk samples of mothers with COVID-19 pneumonia have been tested negative for SARS CoV 2.[12] These reports raise the suspicion of nosocomial transmission to neonates.

Two third of the children were male. It is not clear that the lower incidence of COVID-19 in women is also applicable to female children. Studies suggested that immune regulatory proteins encoded by the $X$ chromosome cause less viral load and inflammation in females than male.[13]

The most common presentation was with gastrointestinal (GI) symptoms. Six patients presented with sudden onset of severe abdominal pain \& several episodes of non-bilious vomiting. Laboratory investigations did not reveal any abnormality rather RT-PCR for SARS Co V2 came positive. One of them had radiological evidence of COVID-19 pneumonia in chest X-ray. No one had respiratory symptoms. All of them improved with conservative treatment. GI symptoms have been reported in up to 10\% of COVID-19 patients.[14] A viral infection is the cause of around 50\% of intussusceptions in children. COVID-19 has also been reported to cause intussusception in children, and pediatric surgeons in an epidemic area should be aware of this.[15-16]

Surgical treatment was required in $14(43.8 \%)$ patients. Others improved with conservative treatment. The personal protective equipment of caregivers was not standard; despite the fact that only seven of the caregivers got COVID-19 during this period. However, it was not certain whether they got the infection from the hospital or outside. Fortunately, all of them improved without complications. The evidence available to date does not support a "child to adult" transmission of SARS Co V2.[17-18]

The mean duration of hospital stay was $5.53 \pm 1.58$ days. No patient had hypoxemia or organ failure. Therefore, intensive care support was not required. This is due to the milder course of COVID-19 even with co-existing surgical emergencies. This finding supports the most published series of COVID-19 in children though serious infection and even death have also been reported in children.[3-6] This might be due to variable viral strains in this region or dissimilar immune responses to it.

Rescheduling of the non-emergent cases has already caused an enormous burden on the health care system. It is still not clear how long this critical period will go on. To reduce the sufferings of children with surgical needs, a gradual restart of scheduled surgeries is necessary considering the low transmissibility of COVID-19 in children.

\section{Conclusions:}

COVID-19 has made the world standstill. It is taking a toll on civilization. Fortunately, it is sparing the children so far but the impact on children's surgery is already huge. Our study has shown the milder course of the disease even with a co-existing surgical emergency in children. The transmission of the disease from children to health care workers was also low. This might encourage the pediatric surgeons around the world for a gradual restart.

\section{Abbreviations}


COVID 19- coronavirus disease 19

SPSS- Statistical Package for the Social Science

IHPS- Infantile hypertrophic pyloric stenosis

ARM- Anorectal malformation

SARS CoV 2- Severe acute respiratory syndrome coronavirus 2

RT-PCR- Reverse transcription polymerase chain reaction

Gl- Gastrointestinal

\section{Declarations}

\section{Acknowledgement:}

In this desperate hour of our history, the heartiest gratitude goes to all those health workers, who are continually fighting risking their lives and saving thousands of lives while at peaked vulnerability.

\section{Contributors:}

Dr. Md Samiul Hasan: Conception, study design, data collection, data analysis, manuscript writing, and submission.

Dr. Md Ayub Ali: Obtaining ethical permission, Data collection, and manuscript revision.

Dr. Umama Huq: Data collection and manuscript editing.

Funding statement: Authors received no fund for this article.

Competing interest: No financial or nonfinancial benefits have been received or will be received from any party related directly or indirectly to the subject of this article.

Ethical approval: Taken from Dhaka Shishu (Children) Hospital ethical committee.

Data availability: All relevant data are included in the manuscript, analyzed raw data are given in the supplementary data. Further specific queries will be entertained by the corresponding author.

\section{References}

1. World Health Organization. Multisystem inflammatory syndrome in children and adolescents temporally related to COVID-19. Available at: https://www.who.int/news$\mathrm{room} / \mathrm{commentaries/detail/multisystem-inflammatory-syndrome-in-children-and-adolescents-with-}$ covid-19. Accessed: 23 July 2020. 
2. WHO Coronavirus Disease (COVID-19) Dashboard. Available at: https://covid19.who.int/? gclid=Cj0KCQjw6uT4BRD5ARIsADwJQ1_hQFnNg35t4jOi8ZrZ_FzvOHOIG_bBbtRmk51v8eSrRC42qM_KMaAgCsEALw_wcB. Accessed: 23 July 2020.

3. Team CC-R. Coronavirus Disease 2019 in Children - United States, February 12-April 2, 2020. MMWR Morb Mortal Wkly Rep. 2020;69(14):422-6. Epub 2020/04/10.

4. Cai J, Xu J, Lin D, et al. A Case Series of children with 2019 novel coronavirus infection: clinical and epidemiological features. Clin Infect Dis.2020. Epub 2020/03/01.

5. Lu X, Zhang L, Du H, et al. SARS-CoV-2 Infection in Children. N Engl J Med. 2020;382(17):1663-5. Epub 2020/03/19.

6. Du W, Yu J, Wang H, et al. Clinical characteristics of COVID-19 in children compared with adults in Shandong Province. China Infection. 2020;48(3):445-52. doi:10.1007/s15010-020-01427-2.

7. Tang D, Tou J, Wang J, et al. Prevention and control strategies for emergency, limited-term, and elective operations in pediatric surgery during the epidemic period of COVID-19. World Journal of Pediatric Surgery. 2020;3:e000122. doi:10.1136/wjps-2020-000122.

8. Al-Jabir A, Kerwan A, Nicola M, et al. Impact of the Coronavirus (COVID-19) pandemic on surgical practice - Part 1. Int J Surg. 2020;79:168-79. https://doi.org/10.1016/j.ijsu.2020.05.022. :ISSN 17439191.

9. Mazingi D, Ihediwa G, Ford K, et al. Mitigating the impact of COVID-19 on children's surgery in Africa. BMJ Global Health. 2020;5:e003016.

10. Keefe $D$, Rickard M, Anderson P, et al. Prioritization and management recommendations of paediatric urology conditions during the COVID-19 pandemic. Canadian Urological Association Journal. 2020;14(6):E237-50. https://doi.org/10.5489/cuaj.6693.

11. Zhoul Y, Xul H, Li L, et al. Management for patients with pediatric surgical disease during the COVID19 epidemic. Pediatr Surg Int. 2020;36:751-2. https://doi.org/10.1007/s00383-020-04656-6.

12. De Rose DU, Piersigilli F, Ronchetti MP, et al. Novel Coronavirus disease (COVID-19) in newborns and infants: what we know so far. Ital J Pediatr. 2020;46:56. https://doi.org/10.1186/s13052-020-0820-x.

13. Conti P, Younes A. Coronavirus. COV-19/SARS-CoV-2 affects women less than men: clinical response to viral infection. J Biol Regul Homeost Agents2020;34(2):10.23812/Editorial-Conti-3. doi:10.23812/Editorial-Conti-3.

14. Flemming S, Hankir M, Ernestus R, et al. Surgery in times of COVID-19-recommendations for hospital and patient management. Langenbecks Arch Surg. 2020;405:359-64. https://doi.org/10.1007/s00423020-01888-x.

15. Martínez-Castaño I, Calabuig-Barbero E, Gonzálvez-Piñera J, et al. COVID-19 Infection Is a Diagnostic Challenge in Infants with lleocecal Intussusception. Pediatr Emerg Care. 2020;36(6):e368. doi:10.1097/PEC.0000000000002155.

16. Moazzama Z, Salim A, Ashraf A, et al. Intussusception in an infant as a manifestation of COVID-19. Journal of Pediatric Surgery Case Reports. 2020;59:101533. 
17. Wongsawat $\mathrm{J}$, Moolasart $\mathrm{V}$, Srikirin $\mathrm{P}$, et al. Risk of novel coronavirus 2019 transmission from children to caregivers: A case series. J Paediatr Child Health. 2020;56:984-5. doi:10.1111/jpc.14965.

18. Posfay-Barbe KM, Wagner N, Gauthey M, et al. COVID-19 in Children and the Dynamics of Infection in Families. Pediatrics2020;146(2): e20201576.

\section{Figures}

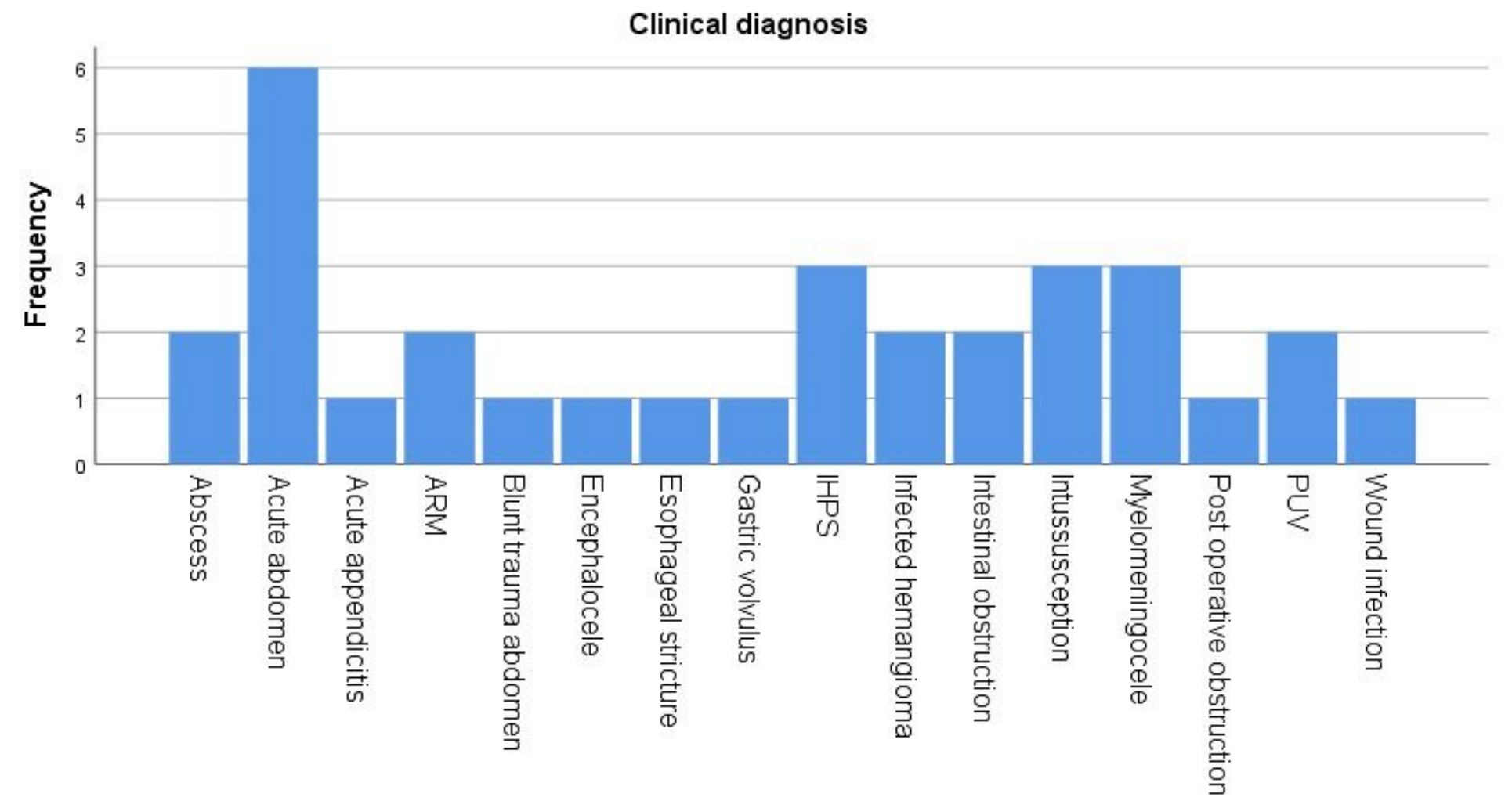

\section{Clinical diagnosis}

\section{Figure 1}

Clinical diagnosis of COVID-19 positive patients.

\section{Supplementary Files}

This is a list of supplementary files associated with this preprint. Click to download.

- supplimentarydata.pdf 